Rev. Elev. Méd. vét. Pays trop., 1970, 23 (1) : 109-17

\title{
Bilan d'un élevage de petits animaux de laboratoire dans certaines conditions tropicales
}

\author{
par G. TACHER \\ (avec la collaboration technique de M. M. ALKADER)
}

\begin{abstract}
RESUME
L'auteur expose les résultats d'un élevage de petits animaux de Jaboratoire: souris, rats, cobayes el lapins conduit dans des conditions africaines à clımat tropical sec sahélo-soudanien.

Du fait surtout de conditions climatologiques trop souvent excessives, morbidité et mortahté sont toujours élevées tandis que la fécondité dans chacune de ces espèces reste sensiblement inférieure à celle observée en régions tempérées.

Seuls des investissements très coûteux pourraient permettre de pallier dans une certaine mesure les effets du climat, avec en tout état de cause une rentabilité très incertanne.

Cela méritait d'être souligné ne serait-ce que pour montrer les difficultés auxquelles se heurtent, en la matière, les chercheurs de laboratorre dans les régions tropicales où le climat n’est favorable à l'élevage des petits animaux d'expérience que 5 mois sur 12 .
\end{abstract}

\section{INTRODUCTION}

La présente note a pour but de définir ce que l'on peut attendre d'élevages de petits animaux de laboratoire: souris, rats, cobayes, lapins en Afrique tropicale à climat sahélosoudanien.

Les statistiques concernant les fluctuations d'effectifs démontrent que les normes couramment admises en Europe ne sont pas extrapolables.

\section{CONDITIONS GENERALES DE L'ELEVAGE}

Dès la mise en service du Laboratoire de Farcha, il a fallu créer un élevage de petits animaux de laboratoire pour effectuer diagnostics et contrôles des vaccins, car aucun de ces petits animaux, souris, rats, cobayes et lapins n'étaient disponibles sur place.
L'élevage a commencé en 1952 et 1953, à partir d'animaux importés du Congo-Brazzaville. Il se heurta à de nombreuses difficultés dont les principales furent le manque de logement, une alimentation mal adaptée et le manque de surveillance.

La construction de locaux, la fabrication et l'achat de cages, le renforcement de la surveillance, la création d'un jardin pour distribution d'aliments verts, la climatisation puis lachat d'une machine à fabriquer les comprimés furent des réalisations qui, effectuées progressivement, permirent d'avoir l'élevage, tel qu'il sera décrit, de 1965 à 1968.

\section{Le climat}

Le climat est caractérisé :

- par une longue saison sèche allant d'octobre à juin. Les températures maximales moyennes mensuelles sont alors de $42^{\circ} \mathrm{C}$ en 
avril-mai, avec un maximum journalier pouvant atteindre $45^{\circ} \mathrm{C}$. Pendant ces mois chauds, la température minimale ne descend pas en dessous de $21^{\circ} \mathrm{C}$. Pendant les mois frais, de décembre à février, la température maximale moyenne varie de 33 à $36^{\circ} \mathrm{C}$ et le minimum absolu enregistré a atteint $8^{\circ} \mathrm{C}$.

L'hygrométrie maximale moyenne varie de 30 à 60 p. 100 et l'hygrométrie minimale moyenne de 10 à 30 p. 100.

- par une saison des pluies s'étalant de juin-juillet à septembre. Les précipitations sont de l'ordre de $600 \mathrm{~mm}$. Les températures maximales moyennes mensuelles varient de $31^{\circ}$ à $35^{\circ} \mathrm{C}$, et la température minimale ne descend pas en dessous de $20^{\circ} \mathrm{C}$.

L'hygrométrie qui remonte dès le mois de mai atteint un maximum en août (l'hygrométrie minimale moyenne est de l'ordre de 30 35 p. 100 et l'hygrométrie maximale moyenne de 65 à 70 p. 100).

La sévérité de ces conditions climatologiques a une influence très déterminante sur l'élevage.

\section{L'habitat}

\section{Les rats et les souris}

Ils sont logés dans un bâtiment de $16 \times 6 \mathrm{~m}$. Les cages sont disposées sur trois rangs, sur un échaffaudage tubulaire.

Les souris sont dans des cages en fer grullagé de $26 \times 21 \times 23 \mathrm{~cm}$ ou en makrolon plein de $30 \times 15 \times 20 \mathrm{~cm}$.

Les rats sont dans les cages en fer grillagé de $30 \times 25 \times 41 \mathrm{~cm}$ ou en makrolon plein de $48 \times 18 \times 28 \mathrm{~cm}$.

Actuellement, les cages en fer sont préférées car bien que les déjections des animaux les détériorent assez rapidement, elles ont le double avantage d'être aérées, ce qui est fondamental lors des grosses chaleurs, et de revenir moins cher car étant fabriquées sur place.

Le local et les cages sont nettoyés tous les jours.

Lorsque la température extérieure atteint $39^{\circ}$, deux climatiseurs de Icv chacun sont mis en route de $10 \mathrm{~h}$. à 18 h., puis fenêtres et portes restent grandes ouvertes. Malheureusement, lorsque la température atteint 44" $\mathrm{C}$, la climatisation ne suffit plus et la température monte dans le local à $41^{\circ} \mathrm{C}$, ce qui provoque une importante mortalité, principalement dans les cages en makrolon qui sont, pour cette raison, abandonnées progressivement.

\section{Les cobayes et les lapins}

Ils sont logés sous un hangar à auvent entièrement ouvert (figure 1), dans des cages à même le sol, cages formées d'une courette de $1,75 \mathrm{~m} \times 1 \mathrm{~m}$ et d'un abri de $0,20 \mathrm{~m} \times 1 \mathrm{~m}$. La nuit, un système de fermeture grillagée (figure 2) permet d'éviter les attaques par les petits carnivores sauvages.

L'élevage comprend 2 rangées de 46 cages séparées par un couloir central.

\section{L'alimentation}

\section{Les rats et les souris}

L'alimentation donnée à volonté est à base de comprimés fabriqués au laboratoire à partir d'aliments locaux. La composition de la ration est la suivante :

\begin{tabular}{|c|c|c|c|c|}
\hline Matières & protéique & brutes & & 20,4 p. 10 \\
\hline Matières & grasses & & & 3,3 p. 10 \\
\hline Cellulose & . & & . & 6,5 p. 100 \\
\hline Glucides & . & . & . & 52,5 p. 100 \\
\hline
\end{tabular}

... Et la formule de l'aliment est :

Petit mil . . . . . . . $3,9 \mathrm{~kg}$

Maïs . . . . . . . $8,2 \mathrm{~kg}$

Gros mil rouge . . . . . $23 \mathrm{~kg}$

Son de blé . . . . . . . $35 \mathrm{~kg}$

Remoulage de blé . . . . 17,5 kg

Farine de sang . . . . . . $9,6 \mathrm{~kg}$

Farine de coquillage. . . . $1 \mathrm{~kg}$

Sel

$\frac{1,8 \mathrm{~kg}}{100}$

Quelquefois, et suivant les disponibilités locales, certains aliments sont supprimés et remplacés par d'autres.

Un peu de lait est donné chaque matin et un mélange polyvitaminé est ajouté à l'eau de boisson qui est donnée à volonté.

Les aliments sont distribués dans des mangeoires posées à même le fond de la cage et la boisson au moyen de biberons suspendus. (Quelques cages ont encore simplement un abreuvoir posé à même le sol). 

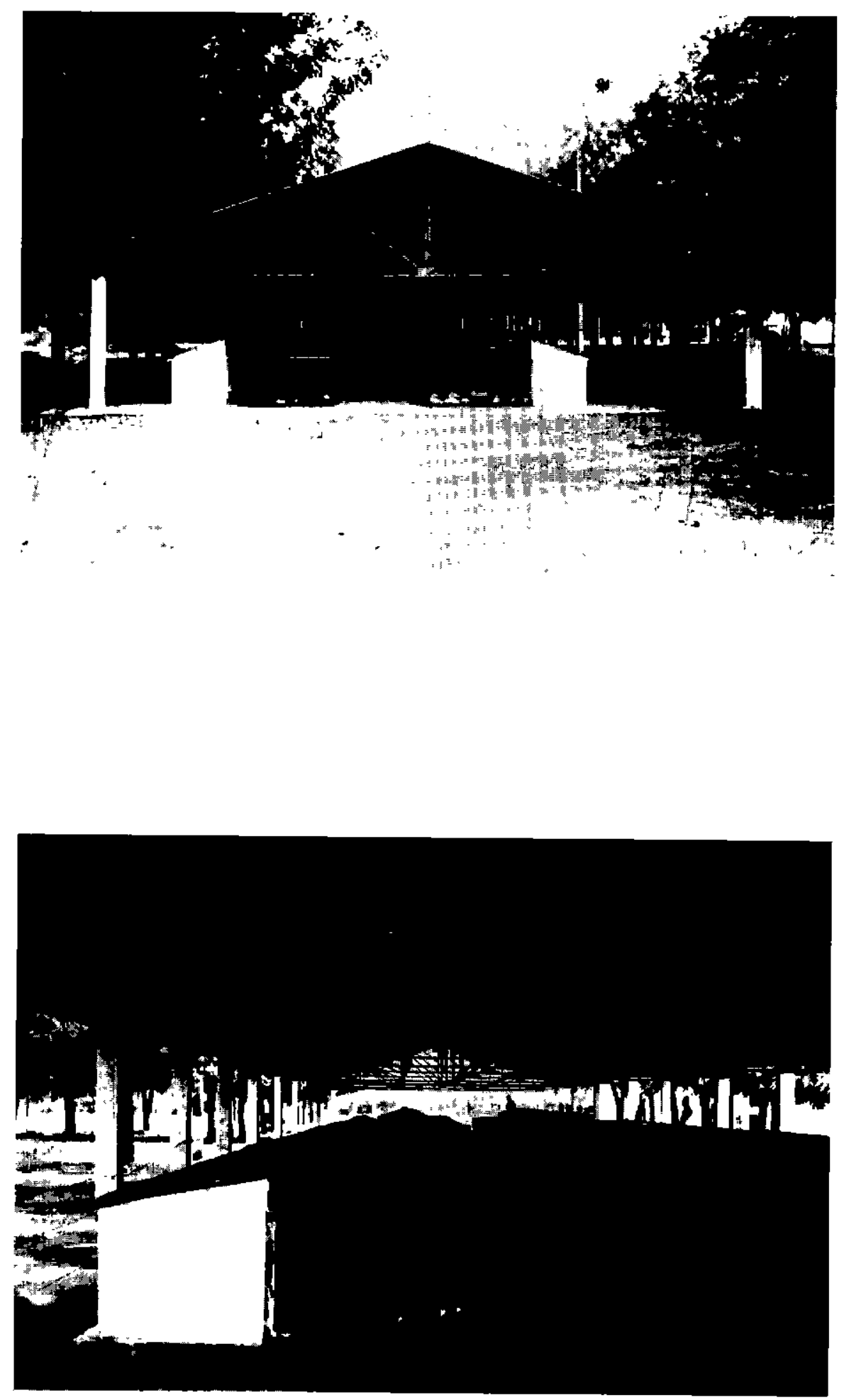
Retour au menu
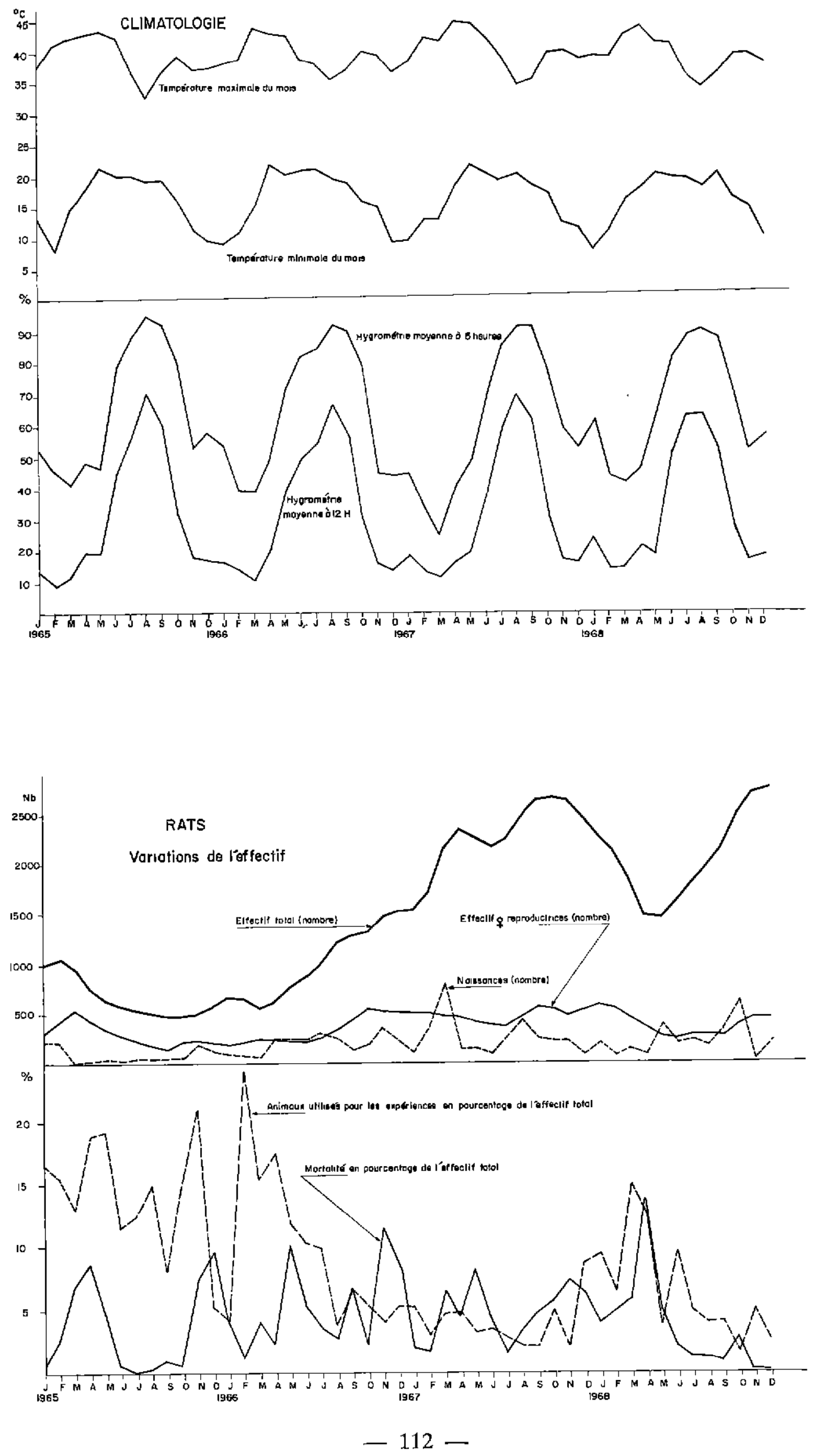


\section{Les cobayes et les lapins}

Les mêmes granulés et le mélange polyvitaminé additionné à l'eau de boisson sont fournis à volonté, ainsi que, tous les matins de l'herbe verte dont la qualité varie selon la saison.

\section{La pathologie}

L'élevage des souris fut décimé en 1958 par l'électromélie. Sa reconstitution fut difficile et il n'a permis de satisfaire les besoins du laboratoire que lorsque la climatisation des bâtiments fut installée. Il est à remarquer toutefois que les souris qui semblaient plus sensibles que les rats à la chaleur avant que la climatisation ne soit installée, paraissent maintenant plus résistantes. Il ne s'agit peut-être là que d'une position dans la pièce par rapport à l'aération.

Depuis 1965, aucun problème spécial de pathologie n'a été relevé dans les élevages de rats et souris. La mortalité importante signalée ne peut être imputée qu'à la chaleur excessive régnant dans les bâtiments, les courbes de mortalités chez ces deux espèces suivant étroitement celles des variations de température et d'hygrométrie.

L'élevage des lapins a à souffrir pendant la saison des pluies et en époque hivernale de pasteurellose. On a dû se résoudre à ne plus utiliser des lapins dans des expériences portant sur les Pasteurella et à fabriquer un auto-vaccin dont la protection s'est révélée faible.

L'élevage des cobayes a eu à subir en 1959 et 1965 des pertes importantes du fait, semblet-il, d'une pneumopathie à ultra-virus analogue à celle décrite par LEPINE, SAUTTER et LAMY.

En 1966, une nouvelle épizootie sévissait, les seuls signes remarqués à l'autopsie étaient ceux de cette même maladie due au virus de la chorio-méningite-lymphocytaire. En 1967, l'élevage se reconstituait. En 1968, une épizootie de salmonellose d'origine inconnue frappait les femelles gestantes, les jeunes et les nouveaunés.

Salmonella typhi-murium fut isolé et un auto-vaccin formolé fut préparé. Son administration par la voie sous-cutanée entraîna de nombreuses mortalités. Ce fut grâce au chloramphénicol administré par voie orale que l'épizootie put être stopée progressivement.

\section{La reproduction}

\section{a) Les rats et les souris}

Les femelles sont mises à la reproduction à l'âge de trois mois à raison d'un mâle pour trois femelles. Elles sont isolées dès les premiers signes de gestation. Les petits sont séparés entre trois semaines et un mois et les mères laissées en repos entre quinze jours et un mois.

\section{b) Les cobayes}

Les femelles sont accouplées à l'âge de trois mois et demi à ratson de un mâle pour trois femelles. Les mâles sont laissés un mois (auparavant, ils n'étaient laissés que huit jours, mais le nombre de femelles non fécondées était très élevé). Les petits sont isolés à un mois. Les femelles sont laissées en repos trois semaines avant d'être remises au mâle.

\section{c) Les lapins}

Les femelles sont couvertes à l'âge de 7 mois. Les lapereaux sont séparés à un mois et demi deux mois. Les femelles sont remises aux mâles

\section{BILAN DE L'ELEVAGE}

Le bilan de l'élevage de janvier 1965 à décembre 1968 est résumé dans les quatre graphiques dans lesquels :

- l'effectif total comprend tous les animaux de l'élevage.

- l'effectif des reproductrices comprend toutes les femelles à compter du moment de la première mise à la reproduction.

- les naissances représentent le total des animaux nés normalement. Il est à remarquer, dans les chiffres qui seront donnés, que, suivant les besoins, les mises à la reproduction pour les rats et les souris peuvent être étalées par rapport aux normes données ci-dessus.

En respectant ces normes toute l'année, on pourrait donc avoir, pour ces deux espèces des taux de naissance supérieurs de 10 à 25 p. 100 à ceux indiqués.

- la mortalité est la mortalité totale, elle englobe donc la morti-natalité.

- Ies animaux utilisés pour l'expérimentation sont des animaux de tous âges et de tous sexes (adultes ou nouveau-nés, aussi bien mâles, les plus nombreux, que femelles comme les rates pour diagnostic de gestation et même que femelles pleines). 

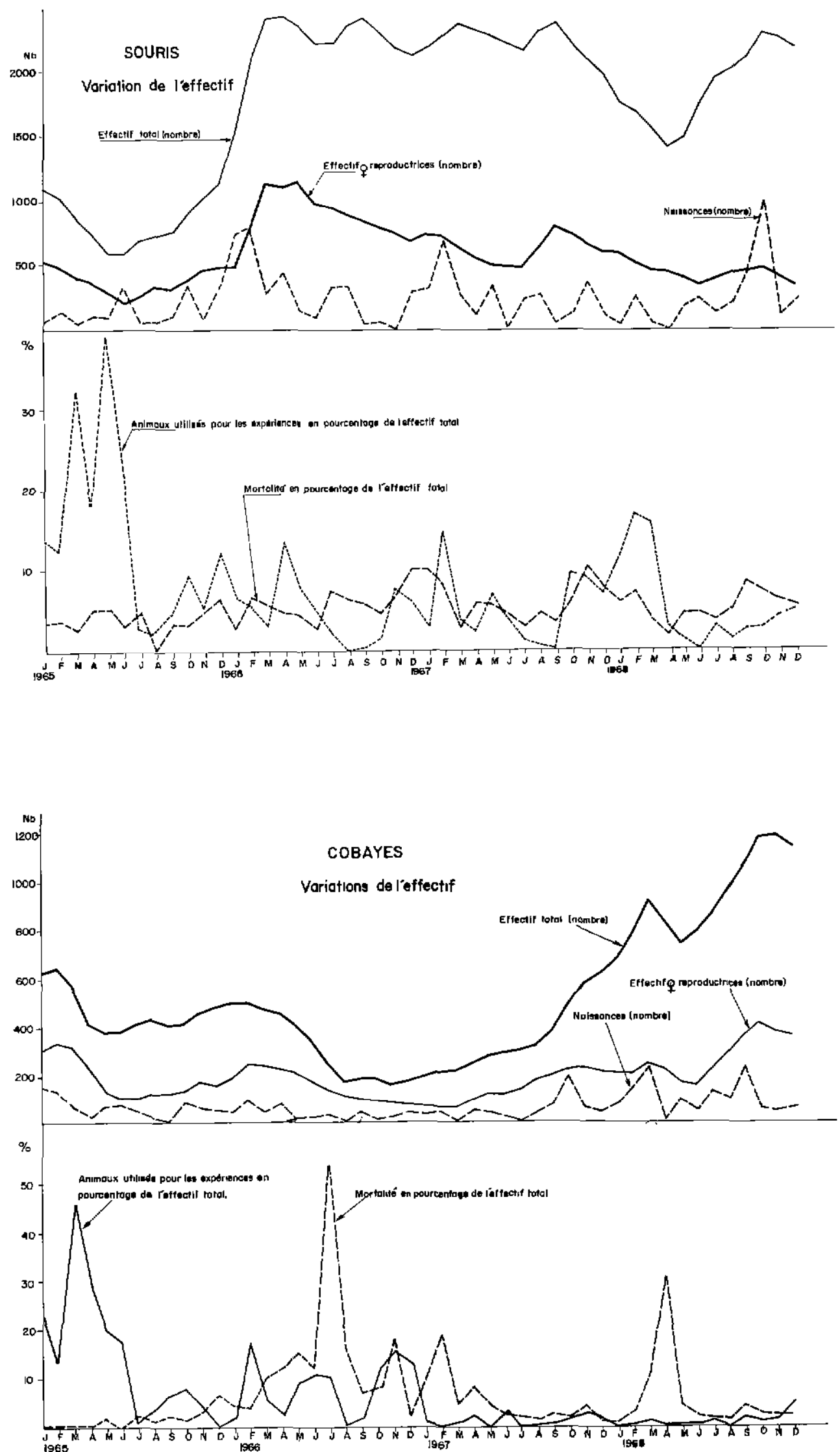


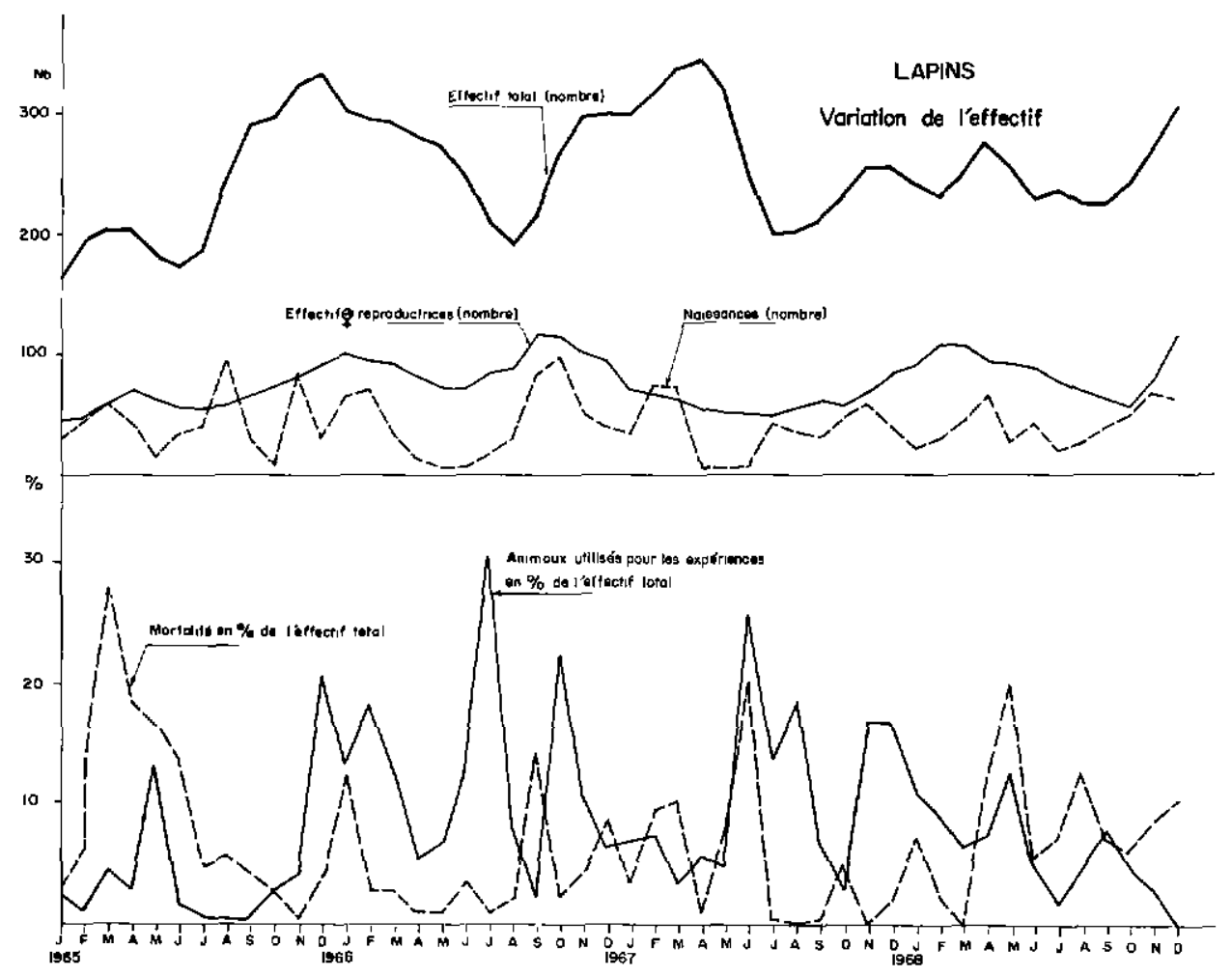

Mode de calcul utilisé

$\begin{array}{llll}1965 \quad 1966 \quad 1967 & 1968\end{array}$

L'effectif moyen total a été calculé ainsi :

- Effectif au premier jour du mois + effectif au dernier jour du mois et division par 2.

- Addition des chiffres trouvés pour chaque mois et division par 12 .

Les mouvements sont calculés de la manière suivante :

- \% pour l'expérimentation =

$$
\frac{\Sigma \text { animaux d'expérience }}{\Sigma \text { effectifs moyens totaux }} \times 100
$$

- \% mortalité $=$

$$
\frac{\Sigma \text { animaux morts }}{\Sigma \text { effectifs moyens totaux }} \times 100
$$

- Chaque femelle donne naissance à X petıts, $\Sigma$ naissances

$\bar{\Sigma}$ effectifs moyen reproductrices

\section{Les rats}

L'effectif au $1^{\text {er }}$ janvier 1965 comptait 994 rats dont 359 reproductrices; au 31 décembre 1968, il comprenait 2.792 animaux dont 454 reproductrices.

Effectif moyen total

Effectif moyen reproductrices

Naissances (total)

Mortalités (total)

Animaux expériences

(total)

$\begin{array}{rrrr}685 & 990 & 2.262 & 2.041 \\ & & & \\ 310 & 342 & 485 & 385 \\ 1.107 & 2.685 & 3.258 & 2.824 \\ 291 & 681 & 1.332 & 788 \\ & & & \\ 1.208 & 1.012 & 1.061 & 1.556\end{array}$

Chaque année sur une moyenne de quatre ans, l'élevage a permis de tirer 80,9 p. 100 de 1'effectif pour l'expérimentation, la mortalité a été de 51,7 p. 100 de l'effectif total et chaque femelle a donné naissance à 6,5 ratons.

\section{Les souris}

L'effectif au $1^{\text {er }}$ janvier 1965 comptait 1.157 souris dont 534 reproductrices; au 31 décembre 1968, il comprenait 2.199 ani-

\begin{tabular}{|c|c|c|c|c|}
\hline & 1965 & 1966 & 1967 & 1968 \\
\hline $\begin{array}{l}\text { Effectif moyen total } \\
\text { Effectif moyen }\end{array}$ & 844 & 2.227 & 2.409 & 1.879 \\
\hline reproductrices & 370 & 876 & 625 & 44 \\
\hline Naissances (total) & 1.787 & 3.564 & 2.955 & 3.32 \\
\hline Mortalités (total) & 409 & 1.569 & 1.641 & 1.269 \\
\hline Animaux expé & & & & \\
\hline (total) & 1.411 & 1.317 & 1.418 & 1.20 \\
\hline
\end{tabular}
maux dont 352 reproductrices. 
Chaque année, sur une moyenne de quatre ans, l'élevage a permis de tirer 72,8 p. 100 de l'effectif pour l'expérimentation, la mortalité a été de 66,4 p. 100 de l'effectif total et chaque femelle a donné naissance à 5,0 souriceaux.

\section{Les cobayes}

L'effectif au $1^{\text {er }}$ janvier 1965 comptait 640 cobayes dont 263 reproductrices; au 31 décembre 1968, il comprenait 1.145 animaux dont 352 reproductrices.

\begin{tabular}{|c|c|c|c|c|}
\hline & 1965 & 1966 & 1967 & 1968 \\
\hline $\begin{array}{l}\text { Effectif moyen total } \\
\text { Effectif moyen }\end{array}$ & 469 & 317 & 342 & 929 \\
\hline reproductrices & 183 & 160 & 144 & 268 \\
\hline Naissances (total) & 86 & 50 & 700 & 1.256 \\
\hline $\begin{array}{l}\text { Mortalités (total) } \\
\text { Animaux expériences }\end{array}$ & 103 & 485 & 179 & 602 \\
\hline (tatal) & 944 & 322 & 65 & 155 \\
\hline
\end{tabular}

Chaque année, sur une moyenne de quatre ans, l'élevage a permis de tirer 72,4 p. 100 de l'effectif pour l'expérimentation, la mortalité a été de 66,5 p. 100 de l'effectif total et chaque femelle à donné naissance à 4,4 petits.

\section{Les lapins}

L'effectif au $1^{\text {er }}$ janvier 1965 comptait 165 lapins dont 40 reproductrices; au 31 décembre 1968, il comptait 323 animaux dont 127 reproductrices.

\begin{tabular}{|c|c|c|c|}
\hline & 1965 & 1966 & 7967 \\
\hline Effectif moyen total & 233 & 266 & 270 \\
\hline reproductrices & 63 & 85 & 61 \\
\hline
\end{tabular}

$\begin{array}{lcccc}\text { Naissances (total) } & 526 & 530 & 460 & 512 \\ \begin{array}{l}\text { Mortalités (total) } \\ \text { Animaux expériences }\end{array} & 295 & 399 & 328 & 254 \\ \quad \text { (total) } & 77 & 155 & 174 & 174\end{array}$

Chaque année, sur une moyenne de quatre ans, l'élevage a permis de tirer 57,7 p. 100 de l'effectif pour l'expérimentation, la mortalité a été de 127,0 p. 100 de l'effectif total et chaque femelle a donné naissance à 6,8 lapereaux.

\section{CONCLUSION}

Les résultats obtenus de ces élevages satisfont actuellement les besoins, malgré tout limités du laboratoire. Si, à l'avenir, un effectif plus important devenait nécessaire, des améliorations, qui ne seraient maintenant économiquement pas rentables, devraient être apportées, elles porteraient en priorité sur une surveillance accrue par du personnel qualifié, ce qui permettrait d'élever le niveau de l'hygiène, de trier et de sélectionner les reproducteurs d'une manière plus poussée. Il n'en reste pas moins que, sous un climat défavorable à l'élevage des petits animaux, les résultats peuvent servir de base à qui voudrait, dans des conditions identiques, monter un élevage de souris, rats, cobayes et lapins.

Institut d’Elevage et de Médecine vétérinaire des Pays tropicaux.

Laboratoire de Farcha, Fort-Lamy (Tchad).

\section{SUMMARY}

Results of a laboratory animals husbandry in some tropical conditions

The author reports the results of a laboratory animals husbandry: mice, rats, guinea-pigs and rabbits set up in african conditions of sahelosudanian dry tropical climate.

On account of too often excessive climatologic conditions, morbidity and mortality are always important whereas the fecundiy remains appreciably lower than that observed in temperate regions.

The climatic effects could be only moderated, to a certain extent, by very expensive investments with in any case a very uncertain rentability.

That points out the difficulties the laboratory research workers are coming up against, in the tropical regions where the climate is propitious to the animals laboratory husbandry only 5 months on 12 . 


\section{RESUMEN}

Resultado de una cría de pequeños animales de laboratorio bajo ciertas condiciones africanas

El autor expone los resultados de una cría de pequeños animales de laboratorio: ratón, rata, conejillo de Indias y conejos realizada bajo condiciones africanas con clima tropical seco sahelo-sudaneso.

Sobretodo a causa de condiciones climatologicas demasiado a menudo excesivas, la morbidez y la mortalidad son siempre elevadas mientras la fecundidad en cada una de dichas especies permanece sensiblemente inferior a la encontrada en regiones templadas.

Solo el invertir muy costoso podria permitir paliar los efectos del clima en un cierto limste, con una rentabilidad muy incierta sea de ello la que fuere.

Era de subrayar eso, para mostrar particularmente las dificuldades con que los investigadores de laboratorio se tropezan a este propósito en las regiones tropicales donde el clima no es favorable para la cria de pequeños animales de experimento sino durante 5 meses de 12 . 